\title{
Short- and Long-Term Effects of a Novel on Connectivity in the Brain
}

\author{
Gregory S. Berns, ${ }^{1}$ Kristina Blaine,, Michael J. Prietula, ${ }^{2}$ and Brandon E. Pye ${ }^{1}$
}

\begin{abstract}
We sought to determine whether reading a novel causes measurable changes in resting-state connectivity of the brain and how long these changes persist. Incorporating a within-subjects design, participants received restingstate functional magnetic resonance imaging scans on 19 consecutive days. First, baseline resting state data for a "washin" period were taken for each participant for 5 days. For the next 9 days, participants read 1/9th of a novel during the evening and resting-state data were taken the next morning. Finally, resting-state data for a "washout" period were taken for 5 days after the conclusion of the novel. On the days after the reading, significant increases in connectivity were centered on hubs in the left angular/supramarginal gyri and right posterior temporal gyri. These hubs corresponded to regions previously associated with perspective taking and story comprehension, and the changes exhibited a timecourse that decayed rapidly after the completion of the novel. Longterm changes in connectivity, which persisted for several days after the reading, were observed in bilateral somatosensory cortex, suggesting a potential mechanism for "embodied semantics."
\end{abstract}

Key words: connectivity; fMRI; reading; resting state

A great book should leave you with many experiences, and slightly exhausted at the end. You live several lives while reading. - William Styron, Conversations with William Styron.

$\mathbf{M}$ OST PEOPLE CAN identify books that have made great impressions on them and, subjectively, changed the way they think. Some can even point to a book that has changed their life. Stephen King, for example, said that Lord of the Flies changed his life, "because it is both a story with a message and because it is a great tale of adventure." Joyce Carol Oates pointed to Alice in Wonderland as "the book that most influenced her imaginative life." It seems plausible that if something as simple as a book can leave the impression that one's life has been changed, then perhaps it is powerful enough to cause changes in brain function and structure. Here, we test this possibility by using functional magnetic resonance imaging (fMRI) to track changes in resting-state brain activity on a daily basis over a period of 3 weeks, during which individuals read a complete novel.

Novels are stories, and stories are complicated objects of communication (Abbott, 2008).* Although several lin-

* "Story" and "narrative" are often used interchangeably. Technically, narrative (vs. expository text) is the representation of events, consisting of a story (as a sequence of events, participating characters, and causal associations linking acts) and discourse (how the story is physically conveyed). Thus, all conveyed stories are mediated by a narrative discourse (e.g., voice, text, video, actors). ${ }^{4}$ guistic and literary theories describe what constitutes a story, neurobiological research has just begun to elucidate brain networks that are active when processing stories. To date, these studies have focused on the immediate response to short stories (Mar, 2011). In other words, current neurobiological theory of stories describes the network of brain regions that is active and presumably responsible for cognitive processing of stories while they are being consumed. While active tasks have traditionally been used to identify functional networks within the brain, resting-state fMRI has become a common tool to identify consistent patterns of correlated activity, termed resting-state networks (RSNs) (Biswal et al., 1995, 2010; Kelly et al., 2012; Raichle et al., 2001).

Cognitive and emotional interventions have been demonstrated to cause transient changes in functional connectivity (Harrison et al., 2008; Hasson et al., 2009; Mackey et al., 2013; Stevens et al., 2010), but it is not known how long these changes last. Some changes appear to be due to transient activation of specific regions, which persists for minutes to hours (Hasson et al., 2009); while others may persist for longer periods of time and may represent cortical reorganization (Mackey et al., 2013). A limitation of these studies that makes it difficult to determine what are short- and long-term changes is the small number of resting-state scans actually performed.

\footnotetext{
${ }^{1}$ Department of Economics, Center for Neuropolicy, Emory University, Atlanta, Georgia.

${ }^{2}$ Goizueta Business School, Emory University, Atlanta, Georgia.
} 


\section{9 nights of reading}
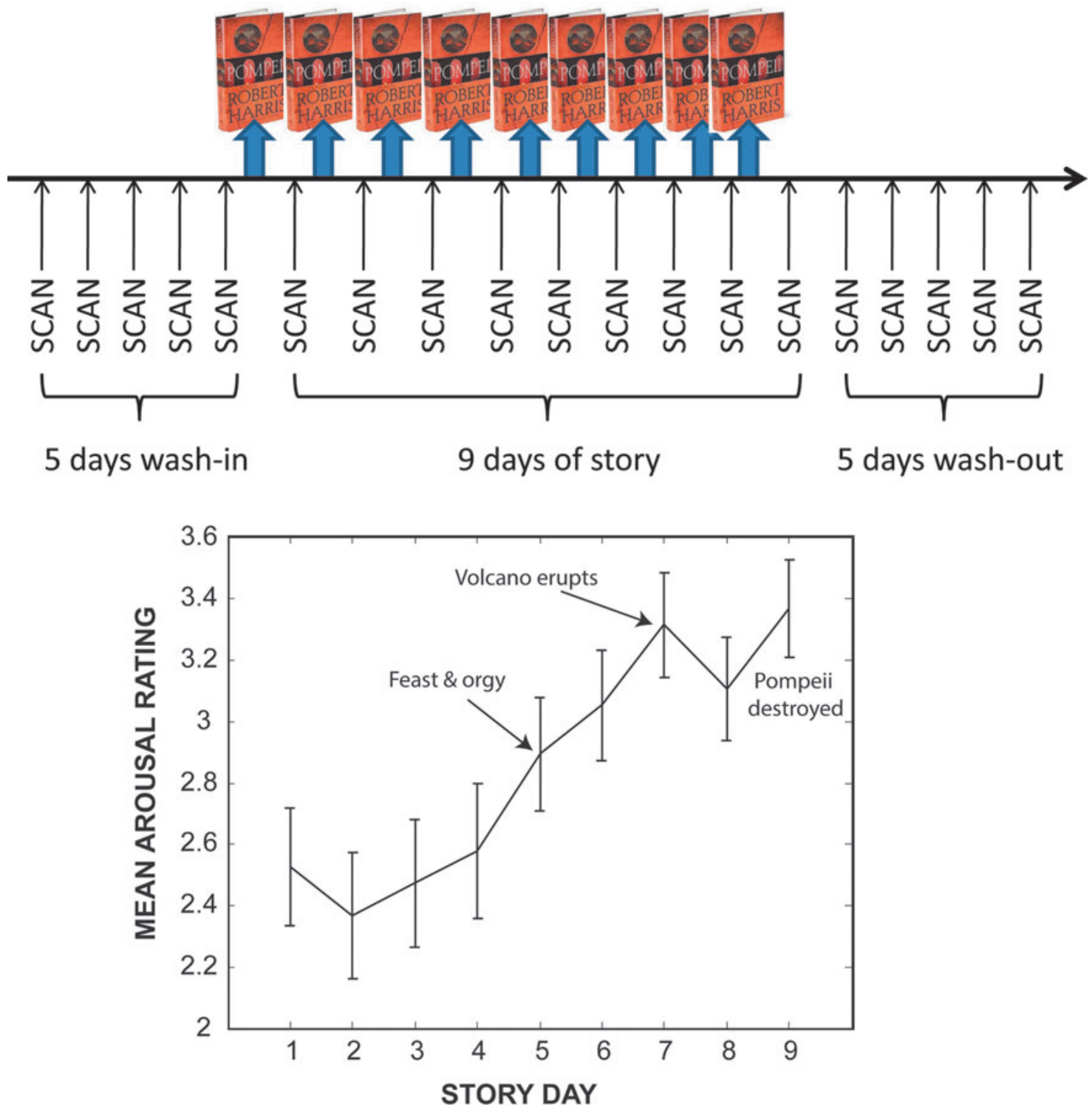

FIG. 1. Design of experiment (above). Participants underwent resting-state functional magnetic resonance imaging scans on 19 consecutive days (black arrows). On the evening before the middle 9 days of scanning, participants read a portion of the novel, Pompeii. The mean arousal rating across participants (below) showed a rising trend toward the climax of the novel (error bars \pm 1 standard error).

To determine a timescale over which connectivity changes persist, we measured changes in resting-state connectivity as a result of reading a novel. We chose a novel over a short story because the length and depth of the novel would afford a set of repeated engagements with associated, unique stimuli (sections of the novel) set in a broader, controlled stimulus context that could be consumed between several scanning periods. A within-subjects design was selected for this pilot study because of its substantive control of individual variability, statistical power, and economic advantages in this type of study (Anderson, 2001; Shadish et al., 2002).

\section{Materials and Methods}

\section{Participants}

A total of 21 participants were studied. Two were excluded from the fMRI analyses: one for insufficient attendance, and the other for image abnormalities. Before the experiment, participants were screened for the presence of medical and psychiatric diagnoses, and none were taking medications. There were 12 female and 9 male participants between the ages of 19 and 27 (mean 21.5). Emory University's Institutional Review Board approved all procedures, and all participants gave written informed consent. 


\section{Reading material}

Each participant was subject to 19 consecutive days (July 18, 2011-August 5, 2011) of resting-state scans that consisted of a total appointment time of less than $30 \mathrm{~min}$ at the same time each day. The first 5 days and last 5 days were "washin" and "washout" sessions, respectively. Each of the middle 9 scans was preceded by reading approximately 1/9th of the novel (Pompeii: A Novel, by Robert Harris, Fawcett, 2003). This novel was chosen because it was based on true events but written as historical fiction and conveyed in a classic narrative arc (Freytag, 1900). During the "washin" and "washout" sessions, the participants did not perform any other tasks except for the resting-state scan (Fig. 1). For each of the other 9 days, the story days, the participants performed the resting-state scan after taking a quiz and self-report about the effect of the material presented in the portion of the novel that was assigned for the previous night and included a five-point rating scale of how arousing the reading was (see Supplementary Data for quizzes; Supplementary Data are available online at www.liebertpub.com/brain). Through repeated scans, each participant served as his or her own control to measure changes in resting-state connectivity after the consumption of the novel.

\section{Scanning}

The scanning was performed on a Siemens 3T Trio. Each participant received only one T1-weighted structural image $\left(\mathrm{TR}=2600 \mathrm{~ms}, \mathrm{TE}=3.93 \mathrm{~ms}\right.$, flip angle $=8^{\circ}, 224 \times 256$ matrix, 176 sagittal slices, and $1 \mathrm{~mm}$ cubic voxel size) throughout the duration of the experiment. One functional resting-state scan was acquired each day $(223$ volumes, $\mathrm{TR}=2000 \mathrm{~ms}, \mathrm{TE}=$ $30 \mathrm{~ms}$, flip angle $=73^{\circ}, \mathrm{FOV}=192 \mathrm{~mm}, 64 \times 64$ matrix, 33 axial slices, and $3 \times 3 \times 3.5 \mathrm{~mm}$ resolution with an added $10 \%$ gap in the $z$-direction, resulting in a resolution of $3 \times 3 \times 3.85 \mathrm{~mm}$ ). Participants were instructed to rest quietly with eyes closed.

\section{Preprocessing}

All of the preprocessing was performed using the 1000 Functional Connectomes Scripts available from NITRC (www.nitrc.org). The only modification to these scripts was the addition of an iterative loop to cycle through the 19days of data. The scripts performed the following preprocessing procedures using FSL (Analysis Group, FMRIB) and AFNI (NIMH). First, the anatomical image was deobliqued and reorientated to the coordinate space that is compatible with FSL. Next, the image was skull stripped.

Table 1. Washout Versus Washin Connections

\begin{tabular}{|c|c|c|c|c|c|c|}
\hline Node & \multicolumn{2}{|l|}{ MNI } & Label & Node & MNI & Label \\
\hline \multicolumn{7}{|c|}{ Washout $>$ washin $(p=0.022)$} \\
\hline 120 & $-6-60-$ & -15 & L cerebellum & 131 & $-34-67-29$ & L cerebellum \\
\hline 130 & $1-66$ & -24 & Cerebellar vermis & 131 & $-34-67-29$ & L cerebellum \\
\hline 109 & $-24-54$ & -21 & L cerebellum & 140 & $33-73-30$ & $\mathrm{R}$ cerebellum \\
\hline 109 & $-24-54$ & -21 & L cerebellum & 150 & $-21-79-33$ & L cerebellum \\
\hline 120 & $-6-60-$ & -15 & L cerebellum & 150 & $-21-79-33$ & L cerebellum \\
\hline 120 & $-6-60-$ & -15 & L cerebellum & 151 & $-6-79-33$ & L cerebellum \\
\hline 130 & $1-66$ & -24 & Cerebellar vermis & 151 & $-6-79-33$ & $\mathrm{~L}$ cerebellum \\
\hline 131 & $-34-67$ & -29 & L cerebellum & 155 & $18-81-33$ & $\mathrm{R}$ cerebellum \\
\hline \multicolumn{7}{|c|}{ Washout $<$ washin $(p=0.003)$} \\
\hline 30 & $\begin{array}{ll}-6 & 17\end{array}$ & 34 & $\mathrm{~L}$ mid cingulate & 43 & $\begin{array}{lll}0 & -1 & 52\end{array}$ & SMA \\
\hline 19 & $-2 \quad 30$ & 27 & $\mathrm{ACC}$ & 103 & $-59-47$ & L middle temporal \\
\hline 30 & $\begin{array}{ll}-6 & 17\end{array}$ & 34 & $\mathrm{~L}$ mid cingulate & 103 & $-59-47 \quad 11$ & L middle temporal \\
\hline 57 & $-12-12$ & 6 & $\mathrm{~L}$ thalamus & 103 & $-59-47$ & $\mathrm{~L}$ middle temporal \\
\hline 58 & $11-12$ & 6 & $\mathrm{R}$ thalamus & 103 & $-59-47 \quad 11$ & $\mathrm{~L}$ middle temporal \\
\hline 61 & $-30-14$ & 1 & L putamen & 103 & $-59-47 \quad 11$ & L middle temporal \\
\hline 43 & $\begin{array}{ll}0 & -1\end{array}$ & 52 & SMA & 109 & $-24-54-21$ & L cerebellum \\
\hline 48 & $-42-3$ & 11 & L insula & 109 & $-24-54-21$ & L cerebellum \\
\hline 62 & $-38-15$ & 59 & $\mathrm{~L}$ pre/post central g & 110 & $-37-54-37$ & L cerebellum \\
\hline 48 & $-42 \quad 3$ & 11 & $\mathrm{~L}$ insula & 113 & $-34-57-24$ & $\mathrm{~L}$ cerebellum \\
\hline 75 & $-38-27$ & 60 & $\mathrm{~L}$ pre/post central g & 113 & $-34-57-24$ & L cerebellum \\
\hline 48 & $-42-3$ & 11 & $\mathrm{~L}$ insula & 120 & $-6-60-15$ & L cerebellum \\
\hline 62 & $-38-15$ & 59 & $\mathrm{~L}$ pre/post central g & 120 & $-6-60-15$ & L cerebellum \\
\hline 75 & $-38-27$ & 60 & $\mathrm{~L}$ pre/post central g & 120 & $-6-60-15$ & L cerebellum \\
\hline 48 & $\begin{array}{ll}-42 & -3\end{array}$ & 11 & $\mathrm{~L}$ insula & 121 & $-25-60-34$ & L cerebellum \\
\hline 31 & $\begin{array}{ll}0 & 15\end{array}$ & 45 & SMA & 128 & $21-64-22$ & $\mathrm{R}$ cerebellum \\
\hline 43 & $\begin{array}{ll}0 & -1\end{array}$ & 52 & SMA & 128 & $21-64-22$ & $\mathrm{R}$ cerebellum \\
\hline 48 & $-42-3$ & 11 & $\mathrm{~L}$ insula & 130 & $1-66-24$ & Cerebellar vermis \\
\hline 75 & $-38-27$ & 60 & L pre/post central g & 130 & $1-66-24$ & Cerebellar vermis \\
\hline 48 & $-42-3$ & 11 & L insula & 131 & $-34-67-29$ & L cerebellum \\
\hline 97 & $-55-44$ & 30 & L supramarginal g & 136 & $-9-72 \quad 41$ & L precuneus \\
\hline 103 & $-59-47$ & 11 & L middle temporal & 136 & $-9-72 \quad 41$ & L precuneus \\
\hline
\end{tabular}

Node is the node number based on the sorting in table S6 by Dosenbach et al. (2010). Network significance is based on 5000 permutations, correcting for FWER. MNI are $x, y, z$ coordinates. Label is based on AFNI "whereami" function and CA_ML_18_MNIA atlas.

FWER, familywise error rate; MNI, montreal neurologic institute; AFNI, analysis of functional neuroimage. 
The resting-state functional images were preprocessed through a multi-step procedure. The images were deobliqued and reoriented similarly to the anatomical images. A mean functional image was computed to serve as a target for motion correction. Using $3 \mathrm{dvolreg}$, the functional images were then aligned to the mean image using two-pass Fourier interpolation. To decrease edge artifacts from Fourier interpolation, a zero pad of four voxels was added around the edges and stripped off after motion correction. The images were then skull stripped to create a mask that was then applied to the motion-corrected data. To allow for full magnetization and settling on any startle responses from the onset of the scanning, the eighth volume was used for registration to the anatomical image. Spatial smoothing was performed using a 6-mm Gaussian kernel. Grand mean scaling was performed with an intensity normalization to 10,000. A lowpass filter of $0.1 \mathrm{~Hz}$ and a high-pass filter of $0.005 \mathrm{~Hz}$ were applied for temporal filtering. The images were detrended by calculating the mean of the temporally filtered image and detrending with the addition of Legendre polynomials of an order up to and including two. An image that was the addition of both the mean and detrended image was created.

Three separate registration alignments were performed. The functional (using the eighth image acquisition as a template) to anatomical alignment was produced using a trilinear interpolation (six degrees of freedom). The anatomical to standard brain (MNI152_T1) was created again using a trilinear interpolation (12 degrees of freedom). The transformation matrices of both of these steps were saved. A third matrix, for the ability to transform between functional to standard, was created by concatenating the matrices of the previous two steps. The inverse of each of these matrices was also produced, providing the option of registering freely between any combination of functional, anatomical, or standard images.

Segmentation was performed to create individual images for each tissue type and individual probability maps. The tissue types recognized as cerebrospinal fluid (CSF) and white matter (WM) were masked. These masks were used to control for nuisance signals. We utilized the global signal, WM and CSF segmentation masks, and the six motion parameters to adjust the functional signals for the effects of physiological noise and motion (Yan et al., 2013). Although adjustment for global signals is controversial, we opted to take a conservative approach and control for physiological noise (Fox et al., 2009). This approach may protect against false positives but may introduce spurious negative correlations (Murphy et al., 2013; Weissenbacher et al., 2009), so our analysis focused only on changes in positive correlations.

\section{Analysis}

Two of the participants were not present on the first day of scanning, and a third was absent on the last day of scanning. Therefore, the 19 participants were analyzed over 17 consecutive days of scanning (days 2-18).

Using a predefined network of 160 regions of interest (ROI) (Dosenbach et al., 2010), we extracted the time series of each ROI for spheres of $6 \mathrm{~mm}$ radius for each person on each day. We chose this set of ROIs because the number of ROIs strikes a balance between a reasonable number and good cortical coverage. Because the original paper was a developmental neuroscience study, our results on reading are particularly relevant to the study of cognitive development. This yielded
L
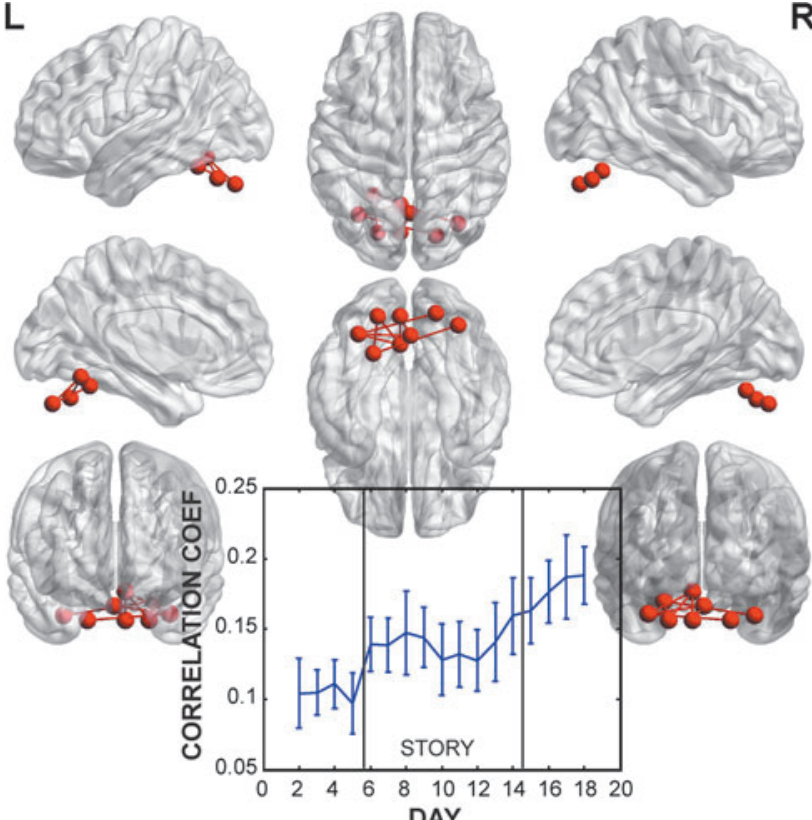

$\mathbf{R}$
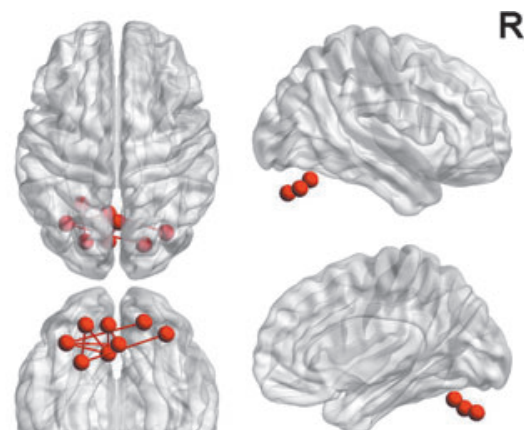

DAY
$\mathbf{R}$
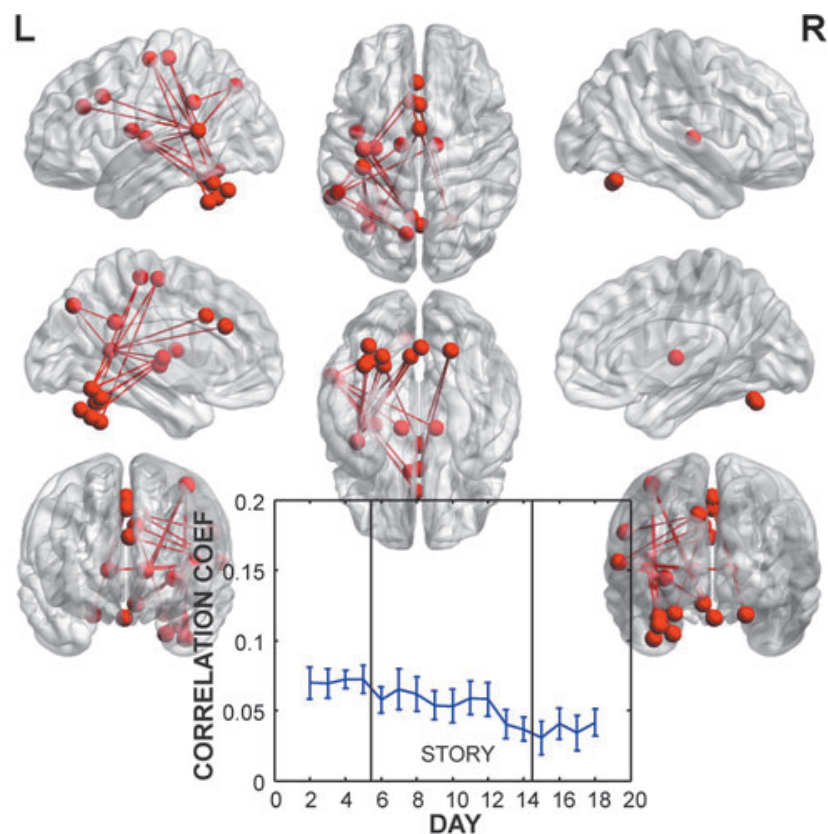

FIG. 2. Networks associated with increased connectivity after the novel [ $p=0.022$ corrected for familywise error rate (FWER)] and decreased connectivity ( $p=0.003$ corrected for FWER). Both networks showed generally monotonic changes in correlation strength with time, suggesting that these changes may not be related directly to the novel itself. It is noteworthy that both networks have strong hubs in the cerebellum. 
a four-dimensional matrix of $\mathrm{ROI} \times$ volume $\times$ person $\times$ day $(160 \times 223 \times 19 \times 17)$. Next, we computed the pairwise crosscorrelation between ROIs for each scan session (Mackey et al., 2013), yielding a matrix with dimensions $160 \times 160 \times$ $19 \times 17$. We then applied the Fisher $z$-transformation to normalize the correlation coefficients, which are bounded by \pm 1 .

Statistics were performed using the Network-Based Statistics (NBS) Connectome v1.2 (Zalesky et al., 2010). All connections in the $z$-transformed correlation matrices were submitted to a one-sided $t$-test to see which individual connections were significantly different based on the specified contrast. The design matrix for these tests included a column for each day and dummy variables for each subject to control for subjectwise differences in mean correlations. Thus, there were 36 columns (17 days +19 subjects). Contrasts were specified as vectors of differences across the 17 day-columns (see below). Due to the large number of elements in the correlation matrix $(12,720$ unique elements), connections that surpassed $p<0.001(t=3.32)$ in significance were then submitted to a permutation test to control for familywise error rate.
NBS is more sensitive to detecting networks of topologically connected nodes, while the related approach-false discovery rate-is more sensitive to strong, focal connections. Since we assume that a novel engages many regions, its effects are likely to be extended over a network of connections rather than a small number of connections. For this reason, we used the NBS intensity statistic. For each contrast, 5000 permutations were performed. Permutations were restricted to be done within subjects only.

Three sets of contrasts were specified. First, to determine whether there were any significant changes in connectivity between the beginning and the end of the story, we examined the contrasts: [washout-washin] and [washin-washout]. Second, to determine short-term changes in connectivity as a result of the story and potential reactivation due to the daily quiz, we contrasted: [story-washin-washout], with appropriate weightings for the different number of days. Finally, to determine long-term changes that were related to the story but persisted beyond the reading days, we contrasted: [story + washout-washin], again with appropriate weightings for the different number of days.

Table 2. Story Versus Nonstory Connections

\begin{tabular}{|c|c|c|c|c|c|c|c|}
\hline Node & MNI & Label & Node & \multicolumn{3}{|c|}{$M N I$} & Label \\
\hline \multicolumn{8}{|c|}{ Network $1(p=0.009)$} \\
\hline 90 & $-8-413$ & L lingual g/hippocampus & 97 & -55 & -44 & 30 & L supramarginal $\mathrm{g}$ \\
\hline 11 & $-11 \quad 4517$ & L sup medial $\mathrm{g}$ & 104 & -53 & -50 & 39 & $\mathrm{~L}$ angular g \\
\hline 13 & $8 \quad 42-5$ & R ACC & 104 & -53 & -50 & 39 & $\mathrm{~L}$ angular $\mathrm{g}$ \\
\hline 17 & $23 \quad 3347$ & $\mathrm{R}$ sup frontal $\mathrm{g}$ & 104 & -53 & -50 & 39 & $\mathrm{~L}$ angular $\mathrm{g}$ \\
\hline 17 & $23 \quad 3347$ & $\mathrm{R}$ sup frontal $\mathrm{g}$ & 107 & 44 & -52 & 47 & $\mathrm{R}$ angular $\mathrm{g}$ \\
\hline 97 & $-55-4430$ & L supramarginal $g$ & 108 & -5 & -52 & 17 & L precuneus \\
\hline 104 & $-53-5039$ & $\mathrm{~L}$ angular $\mathrm{g}$ & 108 & -5 & -52 & 17 & L precuneus \\
\hline 97 & $-55-4430$ & L supramarginal $g$ & 111 & 10 & -55 & 17 & $\mathrm{R}$ precuneus \\
\hline 97 & $-55-4430$ & L supramarginal $\mathrm{g}$ & 115 & -11 & -58 & 17 & $\mathrm{~L}$ precuneus \\
\hline 104 & $-53-5039$ & $\mathrm{~L}$ angular $\mathrm{g}$ & 134 & -36 & -69 & 40 & $\mathrm{~L}$ angular $\mathrm{g}$ \\
\hline 97 & $-55-4430$ & L supramarginal $\mathrm{g}$ & 136 & -9 & -72 & 41 & L precuneus \\
\hline 104 & $-53-5039$ & $\mathrm{~L}$ angular $\mathrm{g}$ & 136 & -9 & -72 & 41 & $\mathrm{~L}$ precuneus \\
\hline 97 & $-55-4430$ & L supramarginal $g$ & 141 & -2 & -75 & 32 & $\mathrm{~L}$ cuneus \\
\hline 97 & $-55-4430$ & L supramarginal $\mathrm{g}$ & 155 & 18 & -81 & -33 & $\mathrm{R}$ cerebellum \\
\hline \multicolumn{8}{|c|}{ Network $2(p=0.012)$} \\
\hline 43 & $\begin{array}{lll}0 & -152\end{array}$ & SMA & 82 & -41 & -37 & 16 & L sup temp g \\
\hline 70 & $42-2417$ & $\mathrm{R}$ sup temp g & 86 & 34 & -39 & 65 & $\mathrm{R}$ post central $\mathrm{g}$ \\
\hline 82 & $-41-3716$ & L sup temp g & 129 & 19 & -66 & -1 & $\mathrm{R}$ lingual $\mathrm{g}$ \\
\hline 64 & $-47-1850$ & $\mathrm{~L}$ post central $\mathrm{g}$ & 145 & -16 & -76 & 33 & L sup occipital g/cuneus \\
\hline 82 & $-41-3716$ & L sup temp g & 145 & -16 & -76 & 33 & $\mathrm{~L}$ sup occipital g/cuneus \\
\hline 89 & $58-4120$ & $\mathrm{R}$ sup temp/supramarginal $\mathrm{g}$ & 145 & -16 & -76 & 33 & L sup occipital g/cuneus \\
\hline 51 & $\begin{array}{lll}46 & -8 & 24\end{array}$ & $\mathrm{R}$ post central $\mathrm{g}$ /insula & 148 & 15 & -77 & 32 & $\mathrm{R}$ cuneus \\
\hline 70 & $42-2417$ & $\mathrm{R}$ sup temp $\mathrm{g} /$ insula & 148 & 15 & -77 & 32 & $\mathrm{R}$ cuneus \\
\hline 89 & $58-4120$ & $\mathrm{R}$ sup temp/supramarginal $\mathrm{g}$ & 148 & 15 & -77 & 32 & $\mathrm{R}$ cuneus \\
\hline 82 & $-41-3716$ & L sup temp g & 156 & -37 & -83 & -2 & $\mathrm{~L}$ inf occipital $\mathrm{g}$ \\
\hline \multicolumn{8}{|c|}{ Network $3(p=0.023)$} \\
\hline 60 & $59-13 \quad 8$ & $\mathrm{R}$ sup temp g & 68 & -54 & -22 & 9 & L sup temp g \\
\hline 68 & $-54-229$ & L sup temp g & 77 & -24 & -30 & 64 & $\mathrm{~L}$ post central g \\
\hline 49 & $-44-649$ & $\mathrm{~L}$ post central $\mathrm{g}$ & 123 & 46 & -62 & 5 & $\mathrm{R}$ middle temp $\mathrm{g}$ \\
\hline 62 & $-38-1559$ & $\mathrm{~L}$ precentral g & 123 & 46 & -62 & 5 & $\mathrm{R}$ middle temp $\mathrm{g}$ \\
\hline 68 & $-54-229$ & L sup temp g & 123 & 46 & -62 & 5 & $\mathrm{R}$ middle temp $\mathrm{g}$ \\
\hline 69 & $41-2355$ & $\mathrm{R}$ pre/post central $\mathrm{g}$ & 123 & 46 & -62 & 5 & $\mathrm{R}$ middle temp $\mathrm{g}$ \\
\hline 75 & $-38-2760$ & $\mathrm{~L}$ pre/post central g & 123 & 46 & -62 & 5 & $\mathrm{R}$ middle temp $\mathrm{g}$ \\
\hline 75 & $-38-2760$ & $\mathrm{~L}$ pre/post central g & 135 & 39 & -71 & 13 & $\mathrm{R}$ middle temp/occipital $\mathrm{g}$ \\
\hline
\end{tabular}

Node is the node number based on the sorting in table S6 by Dosenbach et al. (2010). Network significance is based on 5000 permutations, correcting for FWER. MNI are $x, y, z$ coordinates. Label is based on AFNI "whereami” function and CA_ML_18_MNIA atlas. 
Networks were visualized by displaying nodes and connections in BrainNet Viewer (www.nitrc.org/projects/bnv/). Timeseries for each network were computed by averaging the correlation coefficients in each connection of the network for each subject on each day and then computing the mean and standard error across subjects for each day.

\section{Results}

Consistent with theories of plot structure, the mean arousal ratings of the story rose consistently throughout the story and culminated with the climax-the eruption of the volcano and the destruction of Pompeii (Fig. 1).

For the first set of contrasts, [washout-washin] showed positive correlations that changed significantly between the beginning and the end of the story. This was a small network of eight nodes and eight connections, all in the cerebellum (Table 1 and Fig. 2). The timeseries of this network showed both a monotonic trend throughout most days, but interestingly, the largest increase in correlation was after the first night's reading. During the story days, the correlation fluctuated, but not below pre-story levels, and rose on the last story day with a continued rise after the story. The opposite contrast, [washin-washout] revealed a slow decline in correlations within a network between the left cerebellum and left pre/post central gyrus. These correlations were generally low $(<0.1)$ and decreased to $\sim 0.05$ by the end of the experiment. Given these monotonic trends and preponderance of connectivity changes within cerebellar regions, we do not consider these changes related to the story.

To isolate the short-term changes associated with reading the story, we combined the washin and washout periods and contrasted them with the story days. This contrast identified three independent networks that had significant increases in connectivity during the story days (Table 2). Network 1 had a prominent hub around the left angular and supramarginal gyri with connections to both the precuneus and medial frontal lobe (Fig. 3). There was also a significant connection to the left lingual gyrus in the vicinity of the hippocampus. The timecourse of correlations within this network showed a striking pattern of a sharp rise on the first story day, reaching its peak on the last story day, followed by a nonlinear decay. Network 2 was a bilaterally distributed network without prominent hubs (Fig. 4). Significant connections were mostly posterior and located in the superior temporal gyri and cuneus (Table 2). The timecourse was not as clearly related to the story, with correlations peaking on the second story day and then declining. The correlations were also lower than in Network 1. Finally, Network 3 had significant connections between a hub in the right middle temporal gyrus and the left pre/post central gyrus and left superior temporal gyrus (Fig. 5). The timecourse of correlations of
L
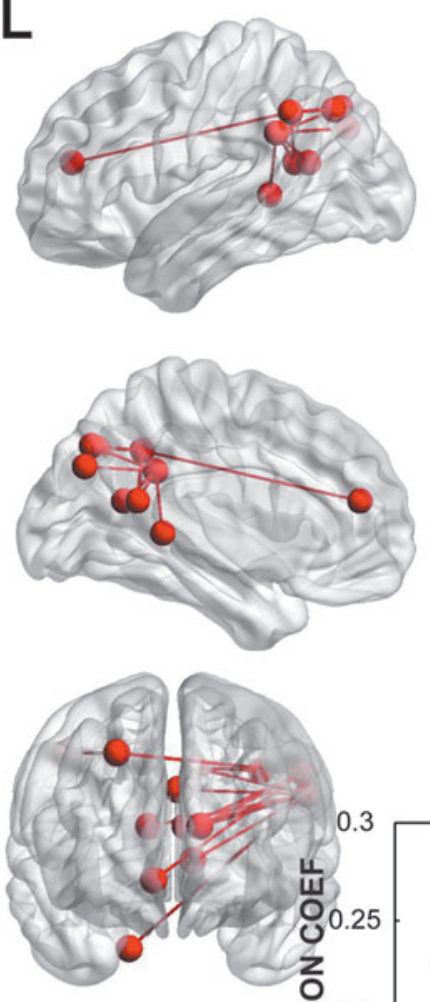
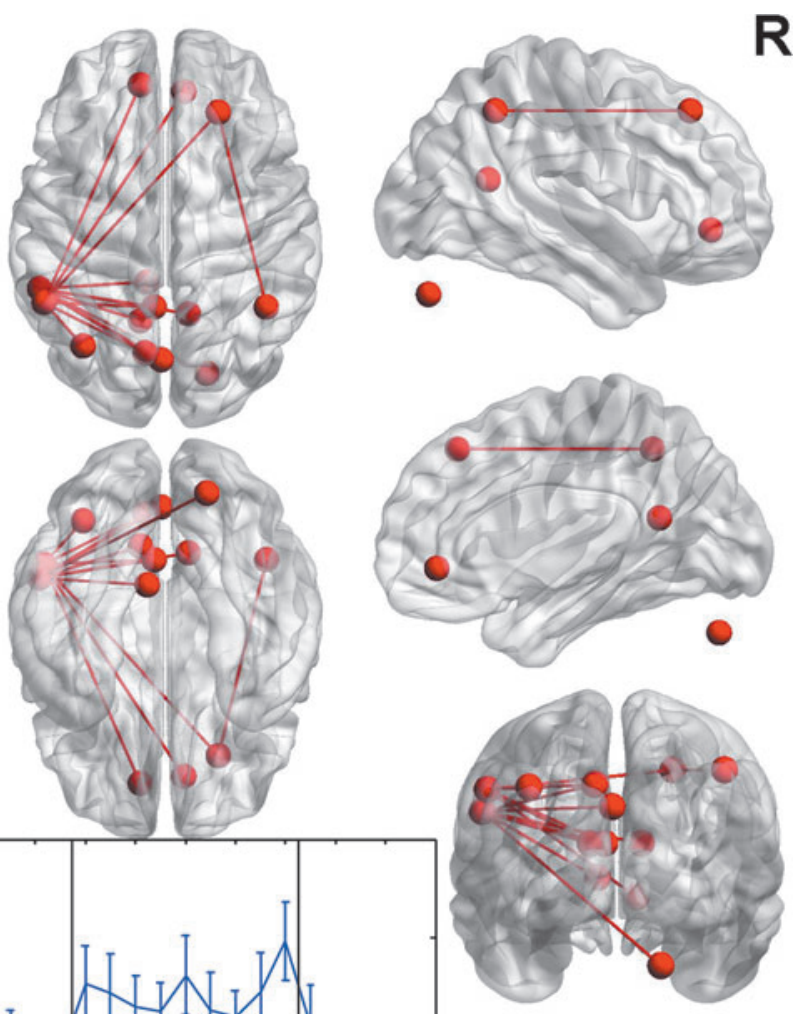

FIG. 3. Network $1(p=0.009$ corrected for FWER) of nodes and connections with significantly increased correlation during story versus nonstory days. This network was concentrated in a hub around the left angular and supramarginal gyri, with connections to medial prefrontal cortex. The timecourse of correlations across days showed a sharp rise beginning on the first post-story day and a decay after the end of the novel. 
FIG. 4. Network $2(p=0.012$ corrected for FWER) of nodes and connections with significantly increased correlation during story versus nonstory days. This sparse network was located in posterior temporal gyri with connections to the cuneus. The timecourse of correlations across days showed a rise beginning on the first post-story and peaking on the second story day, followed by a decline throughout.
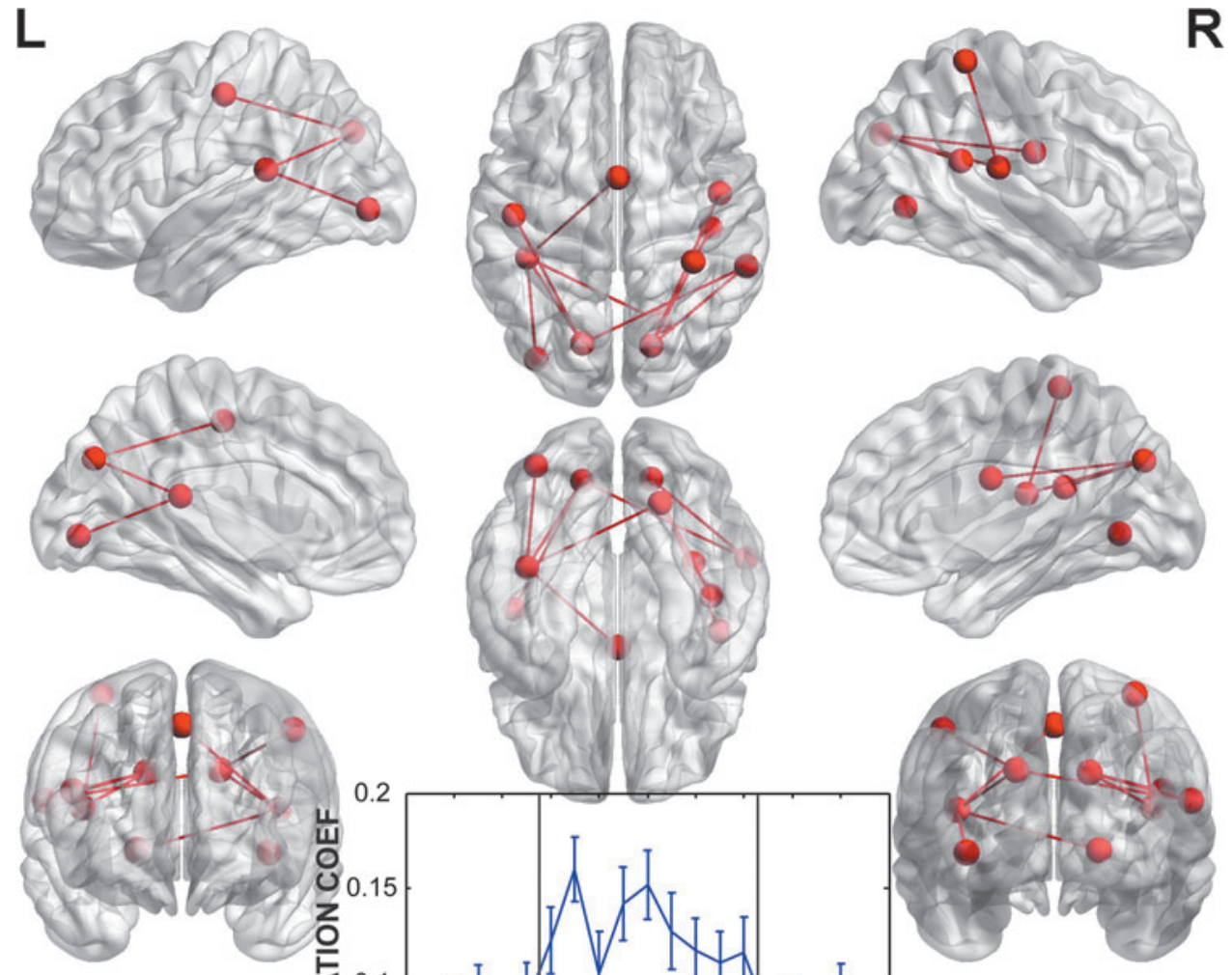

$\mathbf{R}$

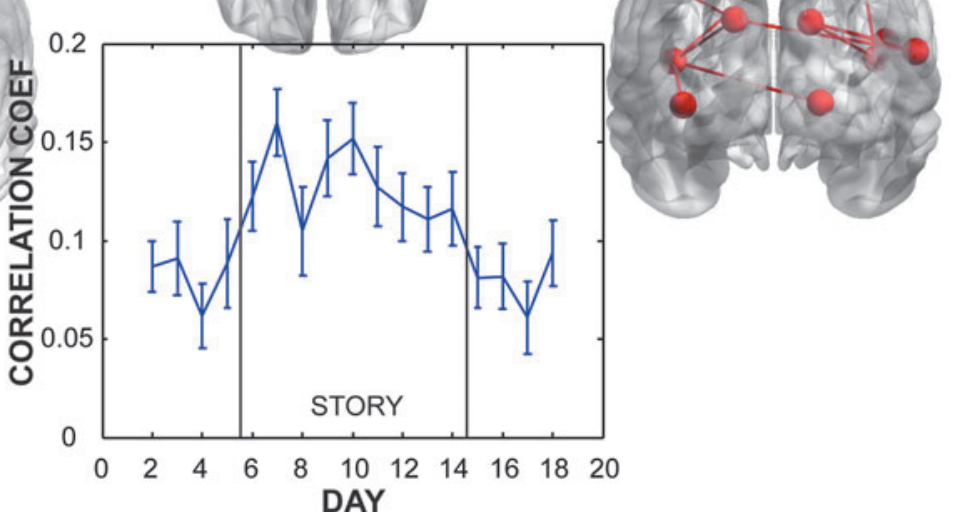

this network showed the same striking increase with the onset of the story. Unlike Network 1, this network did not have a nonlinear decay. The magnitude of correlations were similar to that of Network 1.

To identify potential long-term changes in connectivity, we contrasted [story + washout-washin]. This contrast identified increases in correlation during story days and persisted during the washout period. One network was identified with bilateral connections between pre/post central gyri, middle and superior temporal gyri, and insula (Table 3 and Fig. 6). The timecourse of correlations showed the increase occurring with the onset of the story, peaking on the sixth or eighth story day, and declining slightly afterward. All the correlations during the washout days were higher than the washin period (Fig. 6).

\section{Discussion}

Before interpreting the changes in RSNs, it is worth examining the repeatability of resting-state scans. Previous work has shown that three resting-state scans-two within an hour and one 5-16 months later-demonstrated a modest to high degree of repeatability in the spatial components identified through temporal concatenation independent component analysis (ICA) as well as targeted ROIs (Damoiseaux et al., 2006; Shehzad et al., 2009; Zuo et al., 2010), especially if signals from the CSF were regressed out as nuisance variables (Chang and Glover, 2009; Li et al., 2012). Similar test-retest reliability from temporal concatenation ICA was obtained in older adults scanned twice, 1 year apart (Guo et al., 2012). Measures of theoretical graph connectivity showed moderate test-retest reliability, depending on temporal filter parameters (Braun et al., 2012). Therefore, RSNs are a viable measure of brain network reorganization due to a salient experience, as these networks appear relatively stable and reliable across time in the absence of significant events. This raises the question of whether reading a novel is sufficiently powerful to cause a detectable reorganization of cortical networks.

The timescale of the effect of a novel may be both short and long term. Short-term effects might be observed immediately after reading. For example, RSNs are known to be altered by recent language comprehension tasks (Hasson et al., 2009) as well as visual categorization tasks (Stevens et al., 2010). Although the chapter readings were performed during the evenings before scans, the quizzes occurred just before the scan. The quizzes, therefore, might be responsible for such immediate changes in resting state, though the tasks differ in their orientation. The primary (evening) task involved active consumption of the story, while the next morning the quiz task involved reflection of the story, where the latter 


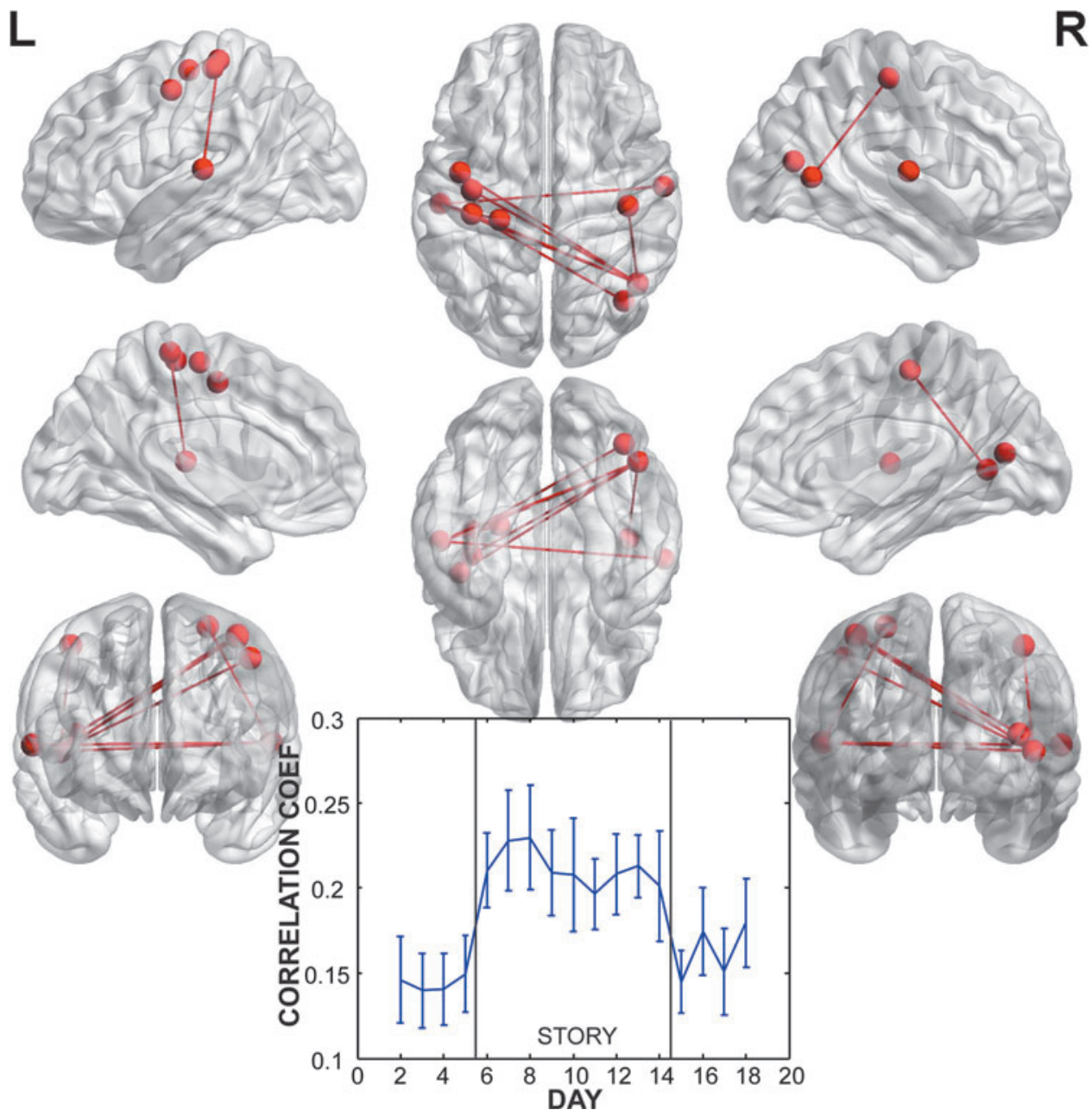

FIG. 5. Network $3(p=0.023$ corrected for FWER) of nodes and connections with significantly increased correlation during story versus nonstory days. This sparse network was located in posterior temporal gyri with connections to the central sulcus. The timecourse of correlations across days showed a sharp rise beginning on the first post-story that was sustained at a relatively constant level throughout the story, followed by a sharp decline post-story. task would likely engage in areas associated with autobiographical recall. The magnitude of the arousal score was not significantly correlated with the changes in connectivity. Even so, recent evidence suggests that resting-state changes persist for a day after a cognitive intervention, such as neurofeedback with a "focusing" effect on loci of activity (Harmelech et al., 2013). Thus, although the reading quiz may be partly responsible for short-term changes in RSNs, there is now evidence that these changes may also be due to carryover from the previous evening.

Considering both the evening carryover and the quiz reactivation as short-term effects, three independent cortical networks demonstrated increases in connectivity as a result of the novel. Network 1 (Fig. 3) displayed the strongest localization to a hub centered around the left angular and supramarginal gyrus with increases in connectivity to both the medial prefrontal cortex (MPFC) and cuneus. The timecourse of correlation within this network displayed a nonlinear decay during the washout period that is consistent with a lingering, but decaying effect. This decay suggests that the changes were not solely due to the quiz, which would have had more of an on-off effect as seen in Network 3 (Fig. 5). The nodal center around the left angular gyrus in Network 1 is consistent with this region's well-known role in language comprehension. A recent meta-analysis of theory of mind studies identified the left angular gyrus as the third most likely region to be acti- vated if the task was story based (behind right angular gyrus and MPFC - both of which also appear in Network 1) (Mar, 2011). Thus, the implication is that the activation of these regions during the evening reading carried over to the next morning as changes in connectivity.

One explanation expands on the concept of resting state as a dynamic organizational construct (Deco et al., 2011). These "resting-state" networks marshal the brain's earlier functional engagement of the novel, particularly the multimodal associative regions around the temporoparietal junction (TPJ) (angular and supramarginal gyri, and middle and superior temporal gyri). This interpretation rests on the principle that the brain is a prediction engine. That is, the resting state of the brain is best viewed as being in a "constant inner state of exploration, in which the brain generates predictions about the likely network configuration that would be optimal for a given impending input" (Deco et al., 2011). Accordingly, since earlier cognitive experiences may modulate the resting-state connectivity maps, the task of reading Pompeii conditionally altered the resting state of our participants with a bias toward a hybrid mentalizing-narrative network configuration even though they were not actively engaged in a task.

Could these specific neural effects be mere consequences not related to story consumption? Variance attributed to the story consumption "treatment" certainly contains an error 
Table 3. Story + Washout Versus Washin Connections

\begin{tabular}{|c|c|c|c|c|c|}
\hline Node & MNI & Label & Node & MNI & Label \\
\hline \multicolumn{6}{|c|}{ Story + washout $>$ washin $(p=0.001)$} \\
\hline 18 & $34 \quad 32 \quad 7$ & $\mathrm{R}$ inf frontal $\mathrm{g} /$ insula & 42 & $43 \quad 112$ & $\mathrm{R}$ insula \\
\hline 53 & $44-1138$ & $\mathrm{R}$ pre/post central g & 62 & $-38-1559$ & $\mathrm{~L}$ precentral g \\
\hline 42 & $43 \quad 112$ & $\mathrm{R}$ insula & 69 & $41-2355$ & $\mathrm{R}$ pre/post central g \\
\hline 43 & $0 \quad-152$ & SMA & 69 & $41-2355$ & $\mathrm{R}$ pre/post central g \\
\hline 49 & $-44-649$ & L post central g & 70 & $42-2417$ & $\mathrm{R}$ sup temp $\mathrm{g}$ \\
\hline 52 & $\begin{array}{lll}-54 & -9 & 23\end{array}$ & $\mathrm{~L}$ pre/post central g & 74 & $18-2762$ & $\mathrm{R}$ pre/post central $\mathrm{g}$ \\
\hline 51 & $\begin{array}{lll}46 & -8 & 24\end{array}$ & $\mathrm{R}$ pre/post central $\mathrm{g}$ & 75 & $-38-27 \quad 60$ & L pre/post central g \\
\hline 70 & $42-2417$ & $\mathrm{R}$ sup temp $\mathrm{g}$ & 75 & $-38-2760$ & $\mathrm{~L}$ pre/post central g \\
\hline 54 & $-47-1236$ & $\mathrm{~L}$ pre/post central g & 76 & $-30-28 \quad 9$ & L insula \\
\hline 74 & $18-2762$ & $\mathrm{R}$ pre/post central g & 76 & $-30-28 \quad 9$ & $\mathrm{~L}$ insula \\
\hline 62 & $-38-1559$ & $\mathrm{~L}$ precentral g & 77 & $-24-3064$ & $\mathrm{~L}$ post central $\mathrm{g}$ \\
\hline 64 & $-47-1850$ & $\mathrm{~L}$ post central g & 77 & $-24-30 \quad 64$ & $\mathrm{~L}$ post central $\mathrm{g}$ \\
\hline 69 & $41-2355$ & $\mathrm{R}$ pre/post central g & 77 & $-24-3064$ & $\mathrm{~L}$ post central g \\
\hline 62 & $-38-1559$ & $\mathrm{~L}$ precentral g & 78 & $51-30 \quad 5$ & $\mathrm{R}$ sup/mid temp g \\
\hline 70 & $42-2417$ & $\mathrm{R}$ sup temp g & 78 & $51-30 \quad 5$ & $\mathrm{R}$ sup/mid temp g \\
\hline 74 & $18-2762$ & $\mathrm{R}$ pre/post central $\mathrm{g}$ & 78 & $51-30 \quad 5$ & $\mathrm{R}$ sup/mid temp g \\
\hline 76 & $-30-28 \quad 9$ & $\mathrm{~L}$ insula & 82 & $-41-3716$ & L sup temp g \\
\hline 64 & $-47-1850$ & $\mathrm{~L}$ post central $\mathrm{g}$ & 83 & $-53-3713$ & L sup/mid temp g \\
\hline 42 & $43 \quad 112$ & $\mathrm{R}$ insula & 95 & $43-43 \quad 8$ & $\mathrm{R}$ sup/mid temp g \\
\hline 82 & $-41-3716$ & L sup temp g & 118 & $-34-60-5$ & $\mathrm{~L}$ inf occipital $\mathrm{g}$ \\
\hline 49 & $-44 \quad-649$ & $\mathrm{~L}$ pre/post central g & 123 & $46-62 \quad 5$ & $\mathrm{R}$ middle temp g \\
\hline 52 & $-54-923$ & $\mathrm{~L}$ pre/post central g & 123 & $46-62 \quad 5$ & $\mathrm{R}$ middle temp $\mathrm{g}$ \\
\hline 65 & $46-2045$ & $\mathrm{R}$ pre/post central $\mathrm{g}$ & 123 & $46-62 \quad 5$ & $\mathrm{R}$ middle temp $\mathrm{g}$ \\
\hline 69 & $41-2355$ & $\mathrm{R}$ pre/post central g & 123 & $46-62 \quad 5$ & $\mathrm{R}$ middle temp $\mathrm{g}$ \\
\hline 64 & $-47-1850$ & $\mathrm{~L}$ post central $\mathrm{g}$ & 145 & $-16-7633$ & L sup occipital g/cuneus \\
\hline 65 & $46-2045$ & $\mathrm{R}$ pre/post central g & 145 & $-16-7633$ & L sup occipital g/cuneus \\
\hline 95 & $43-438$ & $\mathrm{R}$ sup/mid temp g & 145 & $-16-7633$ & L sup occipital g/cuneus \\
\hline 82 & $-41-3716$ & L sup temp g & 156 & $-37-83-2$ & L inf occipital g \\
\hline
\end{tabular}

Node is the node number based on the sorting in table S6 by Dosenbach et al. (2010). Network significance is based on 5000 permutations, correcting for FWER. MNI are $x, y, z$ coordinates. Label is based on AFNI "whereami" function and CA_ML_18_MNIA atlas.

term, but the resultant network components identified are distinctly task specific and revealed across participants rising above the heterogeneous influences of their "off-task" (reading days) as well as "pre-task" (washin period) experiential milieu. It is, however, possible, that the observed changes in connectivity could be due to the overall experimental context of being scanned after reading chapters from a novel-the experiment itself triggers an active process of remembering the previous night's reading, which was further primed by a quiz. Even so, the fact that reading a novel caused changes in cortical connectivity places a bound on the stability of RSNs. While largely stable, the resting state should properly be conceived of as quasi-static and subject to both shortand long-term dynamic reconfigurations.

Longer-term changes in connectivity were identified by the contrast of [story + washout-washin]. This contrast identified connections that increased in strength during the story days and remained elevated after the novel (Fig. 6). This network was heavily concentrated around the central sulcus bilaterally with connections to bilateral posterior temporal gyri and insula. This network corresponds closely to a previously identified RSN comprising somatosensory and motor regions (De Luca et al., 2006). Correlated fluctuations in motor cortex are well known and may not be due to a specific cognitive process (Biswal et al., 1995; Xiong et al., 1999). Thus, we are left with the question as to why these correlations increased with the onset of the novel.
One possibility for increases in somatosensory cortex connectivity is that reading a novel invokes neural activity that is associated with bodily sensations. This is called the theory of "embodied semantics" (Aziz-Zadeh and Damasio, 2008). Somatosensory cortex activation has been previously demonstrated by the reading of metaphors, especially if they are tactile metaphors (Lacey et al., 2012). It is plausible that the act of reading a novel places the reader in the body of the protagonist, which may alter somatosensory and motor cortex connectivity. It is interesting to note, however, that the regions previously called the "protagonist network" - dorsomedial PFC and right TPJ (Mason and Just, 2009)—constitute a very different network than the somatosensory regions and bear more similarity to the networks we identified as having short-term changes. This network can be compared with the predominately cerebellar network identified by the [washout-washin] contrast (Fig. 2). Although the latter represents the most obvious change from before and after the novel, the timecourse is largely monotonic and not clearly related to the novel per se. However, it is possible that both the cerebellar changes and the somatosensory changes reflect changes in motor control related to the act of reading. Such processes might relate to oculomotor coordination and attention, for example, and have nothing to do with the content of the novel.

In summary, we have demonstrated that across the likely array of diverse experiences encountered by our participants, there was a detectable and significant common alteration of 


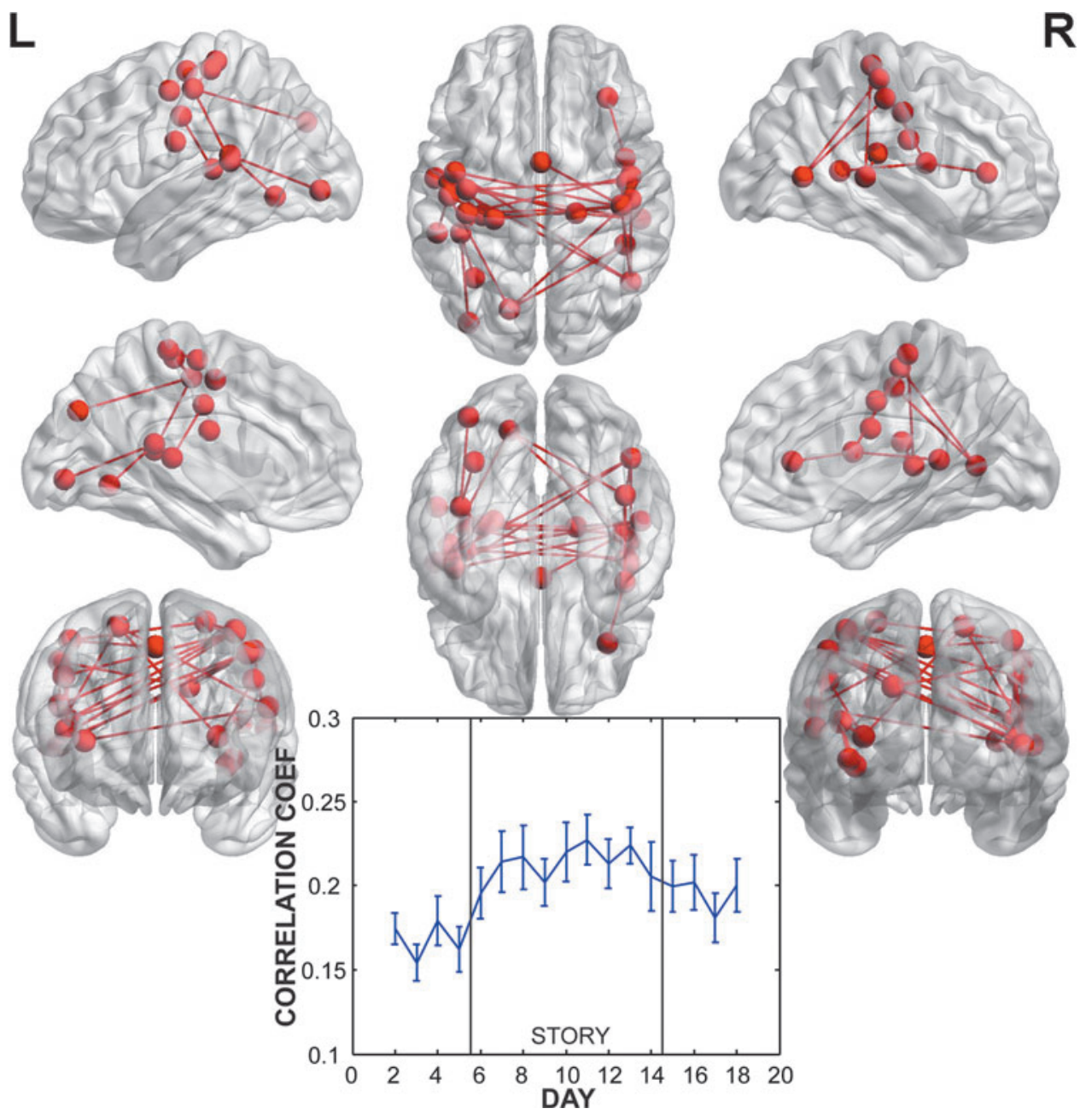

FIG. 6. Network $(p=0.001$ corrected for FWER) of nodes and connections with significantly increased correlation during story days and that persisted beyond the story. This network was located bilaterally around the central sulcus with sparse connections to the insula and occipital regions. The timecourse of correlations across days showed a gradual rise beginning on the first post-story day that was sustained beyond the end of the novel. their RSN associated with reading sections of a novel the previous evening. Moreover, these changes could be segregated into networks associated with short-term changes originating near the left angular gyrus and long-term changes dispersed bilaterally in somatosensory cortex. It remains an open question for further study as to how lasting these effects are, but our results suggest a potential mechanism by which reading stories not only strengthen language processing regions but also affect the individual through embodied semantics in sensorimotor regions.

\section{Acknowledgments}

This research was supported by a grant from DARPA (D11AP00289). The views, opinions, and/or findings contained in this article are those of the authors and should not be interpreted as representing the official views or policies, either expressed or implied, of the Defense Advanced Research Projects Agency or the Department of Defense. This work was approved for Public Release, Distribution Unlimited. The authors are grateful for the input of Andrew Brooks, Lisa LaViers, W. Gavin Ekins, and Ting Li.

\section{Author Disclosure Statement}

No competing financial interests exist.

\section{References}

Abbott HP. 2008. The Cambridge Introduction to Narrative. 2nd Ed., Cambridge: Cambridge University Press.

Anderson NH. 2001. Empirical Direction in Design and Analysis. Mahwah, NJ: Lawrence Erlbaum Associates.

Aziz-Zadeh L, Damasio AR. 2008. Embodied semantics for actions: findings from functional brain imaging. J Physiol Paris 102:35-39.

Biswal B, Yetkin FZ, Haughton VM, Hyde JS. 1995. Functional connectivity in the motor cortex of resting human brain using echo-planar MRI. Magn Reson Med 34:537-541.

Biswal BB, et al. 2010. Toward discovery science of human brain function. Proc Natl Acad Sci USA 107:4734-4739.

Braun U, Plichta MM, Esslinger C, Sauer C, Haddad L, Grimm O, Mier D, Mohnke S, Heinz A, Erk S, Walter H, Seiferth N, Kirsch P, Meyer-Lindenberg A. 2012. Test-retest reliability of resting-state connectivity network characteristics using fMRI and graph theoretical measures. Neuroimage 59:14041412.

Chang C, Glover GH. 2009. Effects of model-based physiological noise correction on default mode network anti-correlations and correlations. Neuroimage 47:1448-1459.

Damoiseaux JS, Rombouts SARB, Barkhof F, Scheltens P, Stam CJ, Smith SM, Beckmann CF. 2006. Consistent resting-state networks across healthy subjects. Proc Natl Acad Sci USA 103:13848-13853. 
De Luca M, Beckmann CF, De Stefano N, Matthews PM, Smith SM. 2006. fMRI resting state networks define distinct modes of long-distance interactions in the human brain. Neuroimage 29:1359-1367.

Deco G, Jirsa VK, Mcintosh AR. 2011. Emerging concepts for the dynamical organization of resting-state activity in the brain. Nat Rev Neurosci 12:43-56.

Dosenbach NUF, Nardos B, Cohen AL, Fair DA, Power JD, Church JA, Nelson SM, Wig GS, Vogel AC, Lessov-Schlaggar CN, Barnes KA, Dubis JW, Feczko E, Coalson RS, Pruett JR, Barch DM, Petersen SE, Schlaggar BL. 2010. Prediction of individual brain maturity using fMRI. Science 329:13581361.

Fox MD, Zhang D, Snyder AZ, Raichle ME. 2009. The global signal and observed anticorrelated resting state brain networks. J Neurophysiol 101:3270-3283.

Freytag G. 1900. Freytag's Technique of the Drama. An Exposition of Dramatic Composition and Art. Chicago: Scott, Foresman and Company.

Guo CC, Kurth F, Zhou J, Mayer E, Eickhoff SB, Kramer JH, Seeley WW. 2012. One-year test-retest reliability of intrinsic connectivity network fMRI in older adults. Neuroimage 61:1471-1483.

Harmelech T, Wertman E, Malach R. 2013. The day-after effect: long term, Hebbian-like restructuring of resting-state fMRI patterns induced by a single epoch of cortical activation. J Neurosci 33:9488-9497.

Harrison BJ, Pujol J, Ortiz H, Fornito A, Pantelis C, Yucel M. 2008. Modulation of brain resting-state networks by sad mood induction. PLoS One 13:e1794.

Hasson U, Nusbaum HC, Small SL. 2009. Task-dependent organization of brain regions active during rest. Proc Natl Acad Sci USA 106:10841-10846.

Kelly C, Biswal BB, Craddock RC, Castellanos FX, Milham MP. 2012. Characterizing variation in the functional connectome: promise and pitfalls. Trends Cogn Sci 16:181-188.

Lacey S, Stilla R, Sathian K. 2012. Metaphorically feeling: comprehending textural metaphors activates somatosensory cortex. Brain Lang 120:416-421.

Li Z, Kadivar A, Pluta J, Dunlop J, Wang Z. 2012. Test-retest stability analysis of resting brain activity revealed by blood oxygen level-dependent functional MRI. J Magn Reson Imaging 36:344-354.

Mackey AP, Singley ATM, Bunge SA. 2013. Intensive reasoning training alters patterns of brain connectivity at rest. J Neurosci 33:4796-4803.

Mar RA. (2011). The neural bases of social cognition and story comprehension. Annu Rev Psychol 62:103-134.
Mason RA, Just MA. 2009. The role of the theory-of-mind cortical network in the comprehension of narratives. Lang Linguist Compass 3:157-174.

Murphy K, Birn RM, Bandettini PA. 2013. Resting-state fMRI confounds and cleanup. Neuroimage 80:349-359.

Raichle ME, MacLeod AM, Snyder AZ, Powers WJ, Gusnard DA, Shulman GL. 2001. A default mode of brain function. Proc Natl Acad Sci USA 98:676-682.

Shadish WR, Cook TD, Campbell DT. 2002. Experimental and Quasi-Experimental Designs for Generalized Causal Inference. Boston: Houghton Mifflin.

Shehzad Z, Kelly AMC, Reiss PT, Gee DG, Gotimer K, Uddin LQ, Lee SH, Margulies DS, Roy AK, Biswal BB, Petkova E, Castellanos FX, Milham MP. 2009. The resting brain: unconstrained yet reliable. Cereb Cortex 19:2209-2229.

Stevens WD, Buckner RL, Schacter DL. 2010. Correlated low-frequency BOLD fluctuations in the resting human brain are modulated by recent experience in category-preferential visual regions. Cereb Cortex 20:1997-2006.

Weissenbacher A, Kasess C, Gerstl F, Lanzenberger R, Moser E, Windischberger C. 2009. Correlations and anticorrelations in resting-state functional connectivity MRI: a quantitative comparison of preprocessing strategies. Neuroimage 47:1408-1416.

Xiong J, Parsons LM, Gao J-H, Fox PT. (1999). Interregional connectivity to primary motor cortex revealed using MRI resting state images. Hum Brain Mapp 8:151-156.

Yan CG, Cheung B, Kelly C, Colcombe S, Craddock RC, Di Martino A, Li Q, Zuo XN, Castellanos FX, Milham MP. 2013. A comprehensive assessment of regional variation in the impact of head micromovements on functional connectomics. Neuroimage 76:183-201.

Zalesky A, Fornito A, Bullmore ET. 2010. Network-based statistic: identifying differences in brain networks. Neuroimage 53:1197-1207.

Zuo X-N, Kelly C, Adelstein JS, Klein DF, Castellanos FX, Milham MP. 2010. Reliable intrinsic connectivity networks: test-retest evaluation using ICA and dual regression approach. Neuroimage 49:2163-2177.

Address correspondence to: Gregory S. Berns

Department of Economics Center for Neuropolicy Emory University 36 Eagle Row Atlanta, GA 30322

E-mail: gberns@emory.edu 